\title{
HANDHELD COMPUTING IN LAW ENFORCEMENT: A PILOT STUDY
}

\author{
András K. Fekete, Andrew L. Kun \\ University of New Hampshire, ECE Department, Durham, NH \{andras.fekete; andrew.kun\}@unh.edu
}

Keywords: Handheld computing, human computer interaction

\begin{abstract}
Handheld computers are being incorporated into many mobile applications. We explore the application of handhelds in law enforcement field operations. We report on a pilot study in which three officers used a handheld computer with the Project54 system.
\end{abstract}

\section{Introduction}

The Project54 system [6] integrates devices in police cruisers into a single system with a speech user interface. Project54 also integrates mobile units (e.g. police cruisers) into agency-wide data networks. The system employs an in-car computer, which runs modular software that in turn controls individual in-car devices and provides the user interface. Currently over 1000 vehicles are equipped with the Project54 system, mostly in New Hampshire.

One problem with the system is that outside the cruiser officers no longer have access to devices in the car, or the data network. To address this problem we have introduced a handheld computer capable of wirelessly connecting to the in-car Project54 computer [4]. Through this connection, the handheld computer can access in-car devices, including the digital radio. Via the digital radio, the handheld can access agency wide data networks. The handheld computers can scan barcodes on driver licenses, and they can use the scanned information to access remote databases to look up driving records. The handhelds can also control in-car devices remotely; e.g. they can turn on lights remotely, which can be useful at accident scenes.

In a pilot program [3] with the New Hampshire State Police three handheld computers, such as the device shown in Figure 1, were deployed with officers in the field. Results from the NHSP deployment indicate that the handheld computer can be useful in law enforcement work. Officers used the handheld to scan driver licenses, operated the in-car radar remotely, etc. Problems were encountered with the volume of the handheld speaker (not loud enough), and with the wireless connection (connection is sometimes lost and user action is required to reconnect). This paper describes a second pilot experiment in which we worked with a local police agency to see how the handhelds suited their needs.

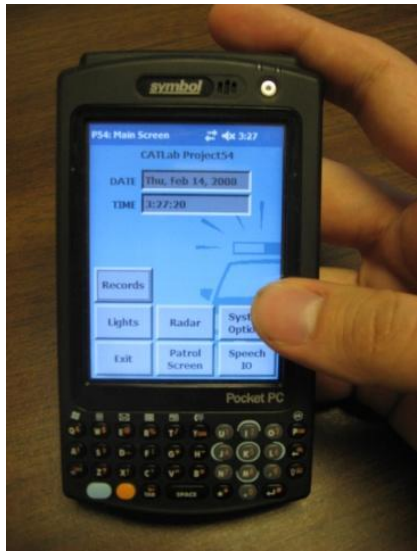

Figure 1: Handheld computer running Project54

\section{Background}

Initial Project54 work on handheld computers involved the development of a remote access system utilizing the Palm Operating System, and a built-in barcode scanner [5]. The Palm prototype system used an external card for scanning and an external radio connection for communication. In later research [4] the system was converted to a handheld running the Windows CE OS. This system contained applications which allowed access to in-car services such as a Radar Application and a Scanner Application. The Windows CE prototype supported the use of speech commands. The prototype also used a CF card 2D barcode scanner to capture license data and an $802.11 \mathrm{~b}$ wireless connection to communicate with the in-car PC.

When writing applications under Windows for PDAs talking to PCs, it is important to consider differences in the .NET framework between the two environments. Roberts and Haddad investigated this issue [9]. Aside from the obvious physical differences between the devices, the two frameworks have subtle differences. There are sometimes function naming differences that necessitate specialized code for each platform. In our case this was not a significant issue as each of the applications are written using a standard library that was created to handle every aspect of the Project54 system (GUI, interapplication communication, etc.).

Handheld-to-PC interactions were explored by the Pebbles project [7]. Applications were developed to enable a handheld to become an extension of a PC. Examples 
include sending keyboard keys, scrolling, menu and toolbar commands, and PowerPoint presentation controls to name a few. Project54 follows this trend, extending the functionality of a police cruiser to a handheld computer.

Myers et al. have also investigated the use of multiple handhelds connected to a PC [8]. This includes applications which allow multiple users to draw simultaneously on a single PC. Applications also allow for users to modify PowerPoint slides, each with his or her own pen. As handheld use becomes commonplace there will be times when multiple handhelds will be present in the same location. In law enforcement we expect this will be the case at accident scenes. In such cases the use of multiple handhelds to share vital information among officers, both on the scene and arriving, could facilitate coordination and efficient use of resources.

In our Project54 system UDP is the communication protocol between the handheld and the in-car computer. Loss of data is a concern, as UDP does not guarantee delivery of the packets transmitted. For this reason Brown and Singh have developed a scheme referred to as M-UDP [1]. M-UDP is designed for mobile devices using a wireless network. The algorithm consists of units acting as Supervisor Hosts $(\mathrm{SH})$ which communicate with a set of Mobile Support Stations (MSS) that talk to Mobile Hosts (MH). Each MSS assigns a special IP address to the MHs it controls so that routing is simple. Information only needs to be handed off when a $\mathrm{MH}$ moves from the control of one $\mathrm{SH}$ to another. The advantage of this approach is that the M-UDP only needs to be implemented at the SH level. Currently Project54 packets transmitted by applications are relatively small, so using a UDP connection is still reliable. Additionally, applications are built to handle dropped messages.

Employing a system relying on a wireless network for data and voice communication is not a trivial task. There are projects however that have successfully used wireless networks in a police setting. The Renton, Washington Police Department, in collaboration with Cisco Systems, set up and used a citywide $802.11 \mathrm{~b}$ wireless network. The network needed to be as secure as their old network and still work with Novell's eDirectory services [2]. Police officers could access this network with wireless devices to get essential information quickly, efficiently, and securely. The network included access to local and national databases. This functionality is also provided by the Project54 in-car system and handhelds.

\section{Deployment steps}

\subsection{System configuration}

We deployed the Project54 handheld computer in the Lee, $\mathrm{NH}$ police department. The handheld was used in a supervisor vehicle that is shared by three officers. The vehicle was a Dodge Charger that had a radar unit, light bar, ODB Diagnostic tool, cellular internet connection (for records queries), a Panasonic ToughBook with Project54 installed and a Linksys USB wireless adapter. We used a Symbol MC50 PDA as the handheld computer (Figure 1).

Communication between the handheld and the in-car PC uses an unencrypted ad-hoc network and all packets transmitted are encrypted using AES encryption with a random key generated for the vehicle. Not all handhelds support the WPA2 standard, the MC50 included, so it was easier to create an open wireless link and implement encryption in our software. For added security the PDA will only accept packets from an IP address that it has been configured to respond to, and the same goes for the PC. These addresses are defined on a per vehicle basis. Therefore, if any other system joins the network, Project54 will not accept traffic from that address.

\subsection{Training}

We spent approximately 10 minutes with one of the three officers explaining the operation of the handheld and the wireless connection. That officer then relayed the information to the other two. Since the officers already were proficient in using the in-car Project54 system, we expected that it would be easy for them to become familiar with the PDA version. This assumption turned out not to be entirely true, which will be discussed in subsequent sections.

\section{Results}

After about a week's use of the system, the officers were asked to fill out a quick survey. The survey inquired about how often they used the handheld and how well they liked certain aspects of the handheld. During the deployment, the handheld was also logging human-computer interactions (button presses and speech utterances).

\subsection{Survey responses}

As with the NH State Police results, the responses given in the surveys were favorable. The survey posed questions about the general characteristics of the PDA, whether it was durable enough, the backlight bright enough, the speakers loud enough, the battery life satisfactory, and so forth. It also asked for opinions on the handheld version of Project54 as a whole, whether the buttons were easy to press, stability of the program, speech recognition performance, and application response time. These items were rated on a ten point Likert scale. Figure 2 shows the median responses to some of the questions. Obviously this is a small dataset, but it gives us some insight into which features work, and which ones need improvement.

Looking at the responses, we see that the biggest problem the officers faced was with the buttons. Specifically, because our deployment happened in the winter, officers used the handheld while wearing gloves and it was difficult to press the small buttons with gloved fingers. One can observe the size of the buttons and an adult 
person's thumb in Figure 1. While using the stylus may have helped with this, the officers did not use it for fear of losing it. Officers were also forced to reset the handheld from time to time due to a bug that was not discovered in lab testing. The officers had some problems with wireless connectivity similar to those we encountered during the NHSP deployment. The officers liked the battery life probably because when the PDA was not in use, it was in the battery charger.

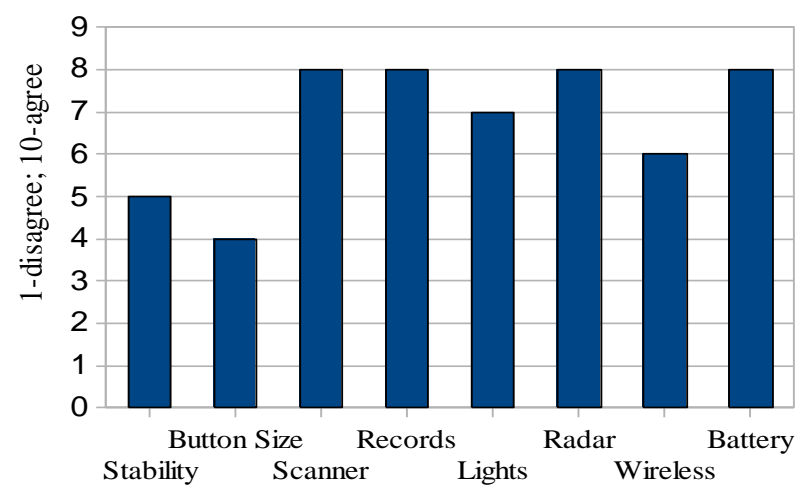

Figure 2: Median responses to survey question results

\subsection{Logging button presses}

We recorded 830 button presses on the handheld. The most frequently pressed buttons had to do with querying remote databases. This is due to the fact that performing a record query requires several steps: selecting the screen for the particular record, scanning a driver license for driver information and finally initiating the query. In addition, the results of a query usually take up multiple screens and officers need to scroll through these screens. In our data scroll buttons were pressed roughly 250 times.

The next most popular set of buttons was part of the Project54 hub application. Switching functionality (e.g. from operating the radar to operating the lights) can only be done by going to the hub application and then selecting the new application. In the hub application the Lights, Records and Exit buttons were most often pressed, 43, 36, and 31 times respectively. Presumably, the Exit button was pressed when shutting down the system at the end of the shift. The other two buttons indicate that officers used (or at least experimented with) the lights and records query functionality.

When performing a query, "Operator by Name" was the most frequently pressed button. This button indicates that the query will use a vehicle operator's name to search for that person's record. The next most frequently pressed button was "Scan." This button activates the barcode scanner. When controlling the lights, "Code One" was the most popular button - this button turns on emergency lights. Officers also used the "Front Antenna" button to operate the front antenna of the radar. Each of these buttons was pressed more than 20 times out of the total 830 presses.
Looking at the button press data, we can conclude that the most popular functionality used by the officers was querying remote databases and operating the lights.

\subsection{Logging speech commands}

Speech input was rarely used on the handheld. About 30 utterances were made during the deployment. Speech was mostly used to switch between application screens. At least one problem with speech input was that officers could not remember which of the five hardware buttons of the handheld to use as the push-to-talk button.

\section{Direct observation results}

We were given the opportunity to observe one officer using the system by joining the officer in the field. This allowed us to better understand handheld usage in the field and it helped us recognize issues that we did not account for in the survey or in our logging.

\subsection{A case of non-intuitive GUI}

Project54 allows for three types of records queries: by name and date of birth, by operator license number (OLN), and by vehicle plate. These query types were designed with speech input to the in-car computer in mind. The idea was that while driving, officers can request data about a car with a specific plate number and when they pull someone over they can request data about a driver by saying the name of the operator or the operator's license number. However, to the officer we accompanied in the field, it did not seem intuitive that on the handheld he must first select a query method and then scan in the license. He expected to be able to scan first and then pick a query method second. However, the laser scanner is only activated when a query method is selected. This resulted in frustration for the officer, since pressing the hardware scan button before selecting the query method had no effect. In future revisions this issue will be resolved by performing a data query using the OLN whenever a license is scanned.

\subsection{Differences between our assumptions and actual use in the field}

Each time the officer pulled someone over, he left the cruiser to approach the driver and ask for license and registration, but not once did he bring the handheld with him as we had hoped. This is because there are several pieces of paperwork to be filled out when pulling over a vehicle, thus carrying the handheld out would not speed things up. Electronic forms could help with this issue.

We were also surprised to see that, to scan the barcode on the back of a license, the officer would hold the license upside down to avoid obscuring part of the barcode with his thumb. Holding the license right side up without the thumb obscuring the barcode required awkwardly pinching the corner of the license, as shown in Figure 3. 
While the orientation of the license and its barcode does not have an effect on system performance, the officer's handling of the license was unexpected for us, and it demonstrates how users will work around problems that crop up with a device used in the field.

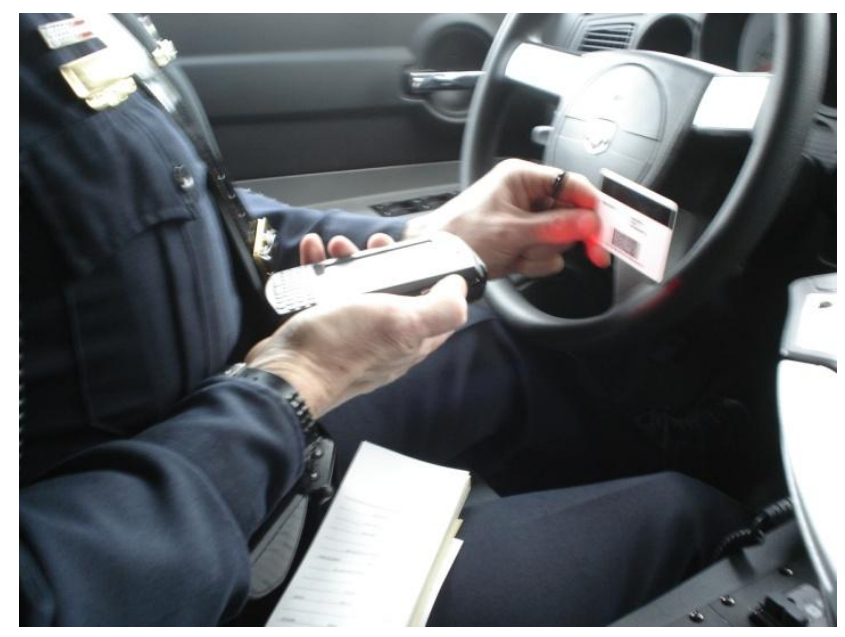

Figure 3: Officer scanning a license.

\subsection{Other thoughts}

Despite all these hurdles, the scanning process worked very well, and on average saved a couple of minutes of typing in the data to perform a record query.

Officers suggested another interesting application for the handheld: a quick check of drinking age. Without the handheld, and its scanner, officers have to take a license and call the dispatcher to verify that the license is valid. With the scanner, a license can be easily verified. Officers also suggested that the age of the licensee should be colorcoded on the handheld display, to indicate whether the person is of drinking age or not.

\section{Future work}

In direct response to the lessons learned from this pilot study, we need to explore the use of larger handheld GUI buttons. We also need to collect more data on the intuitiveness of scanning driver licenses and performing records queries. We need to evaluate how similar the operation of the in-car PC and the handheld need to be so as not to make the process of records queries confusing.

We are also working on a voice and text-messaging feature using a wireless ad-hoc network of handhelds and in-car PCs. This will allow officers at an accident scene to be in communication with other officers at the scene using the program. The feature will allow for sharing pictures taken with the handhelds. Communication will be wireless, and handhelds and in-car PCs will form a mesh network with the ability to relay messages if two devices are not in direct contact.
Another application being tested is using a stand-alone handheld with a cellular connection. This will allow officers who are not in the vicinity of a cruiser, and its digital radio, to access remote databases (e.g. officers on beach patrols, or officers on bicycles).

\section{Acknowledgements}

This work was supported by the US Department of Justice under grant 2006DDBXK099.

\section{References}

[1] K. Brown and S. Singh, "M-UDP: UDP for mobile cellular networks," ACM SIGCOMM Comput. Commun. Rev., vol. 26, no. 5, pp. 60-78, 1996.

[2] Cisco systems, "City of Renton Police Department: Wireless community network protects lives, enhances work," 2005.

http://www.cisco.com/en/US/prod/collateral/wire less/ps5679/ps5861/prod_case_study0900aecd80 306058.pdf

[3] C. Gaudreau, "Project54 handheld system development and pilot deployment." Master's Thesis, University of New Hampshire, 2007.

[4] K. Krstovski, Andrew L. Kun and W. Thomas Miller, III, "Speech interaction with handheld computers in the Project54 system," UbiComp'04, Electronic Adjunct Proceedings, Nottingham, England, September 7-10, 2004.

[5] A. L. Kun and Kadir Dogan, "A prototype remote access and mobile data transaction system for police cruisers," IEEE Spring VTC 2002, Birmingham, AL, May 6-9, 2002.

[6] A. L. Kun, W. T. Miller, III, and W. H. Lenharth, "Computers in police cruisers," IEEE Pervasive Computing, Vol. 3, Issue 4, pp. 34-41, OctoberDecember 2004.

[7] B. A. Meyers, "Using handhelds and PCs together," Communications of the ACM, Vol. 44, Issue 11, pp. 34-41, 2001.

[8] B. A. Myers, H. Stiel, and R. Gargiulo, "Collaboration using multiple PDAs connected to a PC," CSCW, Seattle, WA, November, 1998.

[9] T. Roberts and H. M. Haddad, "Application development for desktop and PDA-style devices: issues and lessons learned," ACMSE 2005, Kennesaw, GA, March 2005. 